\title{
Effectiveness of medical equipment donations to improve health systems: how much medical equipment is broken in the developing world?
}

\author{
Lora Perry $\cdot$ Robert Malkin
}

Received: 16 March 2011/Accepted: 5 May 2011/Published online: 20 May 2011

(C) International Federation for Medical and Biological Engineering 2011

\begin{abstract}
It is often said that most of the medical equipment in the developing world is broken with estimates ranging up to $96 \%$ out of service. But there is little documented evidence to support these statements. We wanted to quantify the amount of medical equipment that was out of service in resource poor health settings and identify possible causes. Inventory reports were analyzed from 1986 to 2010, from hospitals in sixteen countries across four continents. The UN Human Development Index was used to determine which countries should be considered developing nations. Non-medical hospital equipment was excluded. This study examined 112,040 pieces of equipment. An average of $38.3 \%$ (42,925, range across countries: $0.83-47 \%$ ) in developing countries was out of service. The three main causes were lack of training, health technology management, and infrastructure. We hope that the findings will help biomedical engineers with their efforts toward effective designs for the developing world and NGO's with efforts to design effective healthcare interventions.
\end{abstract}

Keywords Healthcare · Technology · Infrastructure · Global health $\cdot$ Medical equipment

Many authors in this publication and elsewhere have dramatically stated that most of the medical equipment in the developing world is broken. Some statements have been vague. In these pages, for example, Bracale and Pepino reported only that "a great deal of equipment is out of order

L. Perry · R. Malkin $(\bowtie)$

Developing World Healthcare Technology Laboratory, Duke University, Hudson 136 \#90281, Durham, NC 27708, USA

e-mail: robert.malkin@duke.edu in most of the health structures in developing countries" [3]. Some have been more precise. For example, Frize and Cheng [7] stated, also in this journal, that up to $60 \%$ of medical equipment is out of service in developing countries. The World Health Organization Guidelines for Healthcare Equipment Donations states that $70 \%$ of medical equipment in sub-Saharan Africa is out of service. The Director General of the World Health Organization stated at the Medical Device Meeting in the fall of 2010 that "about $70 \%$ of the more complex medical devices do not function when they reach their destination." Other papers state that up to $96 \%$ of medical equipment is out of service $[4,11,14-16]$.

While the shock factor of such statistics is great, the scientific value is limited because they have rarely been supported with verifiable data. We have analyzed data from over 100,000 pieces of medical equipment in resource poor settings. The facts do not support the most dramatic claims. In fact, on average, only about $40 \%$ of medical equipment in resource poor settings is out of service.

The only verifiable way to determine what equipment is working is to take an inventory. There are only a few published studies that have documented their inventory methods. We examined original inventory data from fifteen countries spanning the Americas, Africa, and Southeast Asia over the years 1986-2010 [2, 5, 6, 8, 12, 13]. For each inventory, we considered the country to be a developing nation if it was ranked as having "Low" or "Medium" human development by the UN Human Development report (UN Human Development Index, UNHDI) in the year the inventory was taken or, for those countries that were studied before the UNHDI was created, the 1991 UNHDI. We only included inventories that stated that they were exclusively inventorying medical equipment, or where we could remove the non-medical equipment. 
Table 1 Data from 16 countries showing numbers of medical equipment pieces out of service

\begin{tabular}{lllll}
\hline Year & Country & Total pieces & \# Out of service & $\begin{array}{l}\text { Percent out } \\
\text { of service }(\%)\end{array}$ \\
\hline 1986 & Belize & 50 & 20 & 40.00 \\
1986 & Costa Rica & 962 & 8 & 0.83 \\
1986 & El Salvador & 1,384 & 353 & 25.51 \\
1986 & Guatemala & 931 & 165 & 17.72 \\
1986 & Honduras & 785 & 122 & 15.54 \\
1986 & Nicaragua & 1,065 & 310 & 29.11 \\
1986 & Panama & 829 & 59 & 7.12 \\
1987 & Bolivia & 3,296 & 1,335 & 40.50 \\
1987 & Colombia & 14,962 & 6,817 & 45.56 \\
1987 & Ecuador & 3,405 & 1,390 & 40.82 \\
1987 & Peru & 4,253 & 1,844 & 43.36 \\
1987 & Venezuela & 6,527 & 3,068 & 47.00 \\
1991 & Nigeria & 2,199 & 674 & 30.65 \\
2005 & Indonesia & 2,365 & 428 & 18.10 \\
2008 & Ethiopia & 65,668 & 25,610 & 39.00 \\
2010 & Cambodia & 3,359 & 722 & 21.49 \\
Total & & 112,040 & 42,925 & 38.31 \\
\hline
\end{tabular}

Most of the inventory reports included an introductory section. We attempted to extract and compile reasons for the equipment being out of service from these introductions. Using this method, the reasons could be extracted but the numbers of affected pieces could not.

In total, we compiled data for 112,040 pieces of medical equipment. Ninety-three percent of that equipment was from the developing world (Table 1). In developing world countries, $38.3 \%$ (42,925 pieces) of the equipment was out of service.

The percent of equipment out of service in individual countries varied from less than 1\% (Costa Rica) to $47 \%$ (Venezuela). We found no documentation to support reporting more than $50 \%$ (rounding up) of medical equipment out of service.

Sorting the inventories by equipment type does not reveal a strong pattern (Fig. 1). Nevertheless, some equipment was more likely to be out of service (X-ray and sterilizers) while some was less likely to be found out of service (anesthesia machines and operating room tables). Individual types of equipment were out of service at a rate of $32 \%$ (anesthesia machines) to $47 \%$ (X-ray machines).

Analyzing the introductions to the inventory data, we found that the state of the equipment was dependent on factors beyond the equipment itself in three major categories: infrastructure and resources, health technology management (HTM), and training.

Infrastructure and resource deficiencies are system-wide deficiencies. Commonly cited causes for out of service equipment in this category included the lack of spare parts, lack of disposables and lack of required accessories [2, 6, 8, 9].

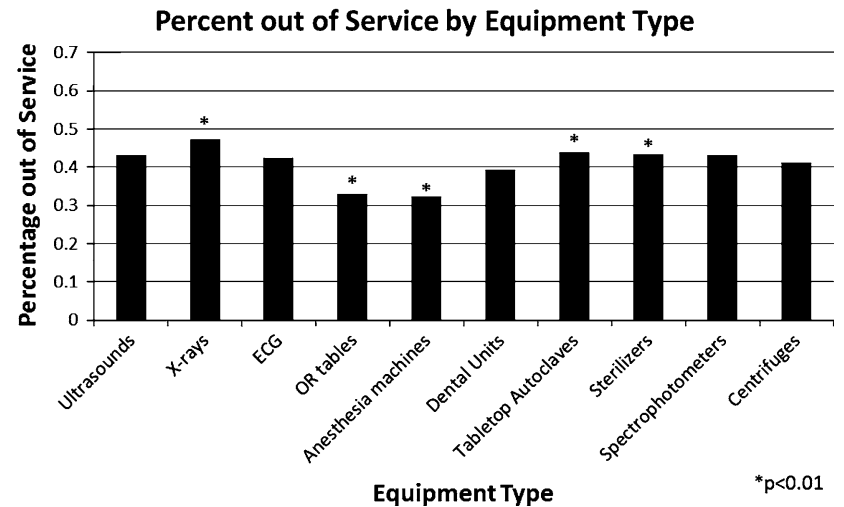

Fig. 1 Percent of equipment out of service by type. Significant variance from the mean is indicated by an asterisk (Cambodia data not included)

HTM problems were also common. Regardless of fault, users sometimes failed to report equipment problems to technicians or administrators [2]. Regular preventative maintenance schedules were rarely followed $[2,6,8,12,13]$ leading to early breakdown and escalation of problems. In most systems, administrators or donors were left making procurement decisions without technical advice $[2,9,12$, 13]. Adding to the systemic burden of non-functional equipment is the fact that many hospitals reported no system for disposal of irreparable or outdated devices $[2,6,8]$.

Capacity building is frequently considered in the medical ranks, but it is also a problem with medical equipment. However, technicians are not the only ones who should understand their technology. Users, administrators, and 
donors need a base level of knowledge. In many systems, users were not trained in the proper use or handling of equipment, leading to avoidable break downs [2, 6, 8, 9]. When technicians were available to attempt repairs or maintenance, there was often a gap between their knowledge and the level of technology [8, 13]. Unfortunately, technicians with even minimal training were reported to be rare. More than half of the technicians, and even maintenance department heads in some regions, were not formally trained biomedical technicians $[2,13]$. Increased capacity must be coupled with increased repair infrastructure. One aspect of repair infrastructure is the service and operator manuals, with at least $50 \%$ of each type not found in the surveyed health systems $[2,8]$.

Compounding the knowledge problem is the fact that most of the equipment comes from outside of the country. Not all of the inventories listed the manufacturer. However, in a subset of 1,242 pieces from 10 hospitals in Indonesia, only 52 pieces $(4.2 \%)$ of the medical equipment were produced in-country. This is quite close to the $5 \%$ reported by Banta [1] in the case of Brazil in the 1980s. Wheelchairs (11) and lighting devices (including exam, viewers and surgery, 11) made up the largest category of locally produced medical equipment. Tables (exam and surgery, 7), sterilizers (steam and hot water, 9), and scales (5) were the majority of the remaining locally produced pieces. Imported equipment came mostly from China, Japan, the United States, and Europe.

If about $40 \%$ of donated medical equipment is out of service, does a donation strengthen the health system? The answer probably depends on the specific piece that is being donated. As we have stated, the amount of out of service equipment does not depend strongly on the type of equipment. We can conclude that about $40 \%$ of donated $\mathrm{X}$-ray machines and about $40 \%$ of donated sphygmomanometers do not work. However, a given donation may provide 100 sphygmomanometers while only providing one X-ray machine. In this case, the donation of sphygmomanometers undoubtedly would help. However, there is about a $40 \%$ chance that the X-ray equipment donation would not.

There are many complicating factors in the interpretation of this presentation. For example, we report that a commonly cited cause for equipment being considered out of service is the lack of spare parts and disposable accessories $[2,6,8,9]$. However, in one of the very few prospective studies, Malkin and Keane [10] showed that $66 \%$ of out of service equipment (of about 3,000 pieces studied) could be returned to service using only locally available materials and less than $\$ 50$. This suggests that there may be a predisposition to designating a piece of equipment as being out-of-service. Or, this could indicate that the higher level of training of most of the volunteers in the Malkin and
Keane study allowed for more repairs. Their theory is currently being prospectively studied in Rwanda.

Clearly one component that is discussed very little in the reviewed inventories is the proportion of inappropriate donations. During the Bangkok Medical Device meeting mentioned above, the WHO Director General suggested that "only 10 to $30 \%$ of donated equipment ever becomes operational". While the vast majority of donations are given with the genuine intent to strengthen the health system, probably a large percentage did not consider the technical infrastructure of the receiving hospital. Medical equipment is designed to operate where there is stable electricity and sophisticated amenities such as purified water or pressurized gas. Such infrastructure is rare [2, 6]. Drastic environmental changes also pose a challenge to operation and maintenance of modern, delicate equipment [6]. Even American hospitals often rely on medical service contracts to keep their equipment running. From the analysis, it is apparent that many organizations, even those donating new equipment, are doing so without donating a service contract $[2,13]$. In many cases, a donation without a service contract very quickly leaves the hospital with an inoperative piece of equipment and a disposal problem. In other words, the donation leaves the hospital worse off.

The analysis may underestimate the number of pieces out of service. We have relied on hospital reported equipment status reports to create the analysis. However, hospitals may ignore some working equipment that never entered their inventory, broken equipment off inventory or they may lack the technical staff to conduct a thorough inventory. In any case, we suspect that many hospitals are underreporting their broken equipment. Unfortunately, we are aware of no independent, peer-reviewed study that tracks a particular shipment of donated equipment from original status to final disposition.

So, how much equipment is out of service in the developing world? The answer is about $40 \%$. We find no evidence to support the statement that most or nearly all the equipment in resource poor settings is broken.

While donations can cause problems and there are undoubtedly some hospitals where the situation is much worse, the analysis does suggest that donating equipment can improve the available healthcare options.

On the other hand, investments in capacity building, health technology management and infrastructure could nearly double the amount of working medical equipment without the expense of collecting, testing, and shipping used medical devices.

Acknowledgments This study was partially funded by the World Health Organization, Essential Healthcare Technology group. We would like to thank International Aid for providing the inventory data for Indonesia and Engineering World Health for providing the inventory data for Cambodia. 


\section{References}

1. Banta HD (1986) Medical technology and developing countries: the case of Brazil. Int J Health Serv 16:363-373

2. Bekele H (2008) Assessment on medical equipment conditions. Ethiopian Science and Technology Agency Ethiopian Scientific Equipment Center, Addis Ababa

3. Bracale M, Pepino A (1994) Medical technologies in developing countries: a feasibility study on the maintenance of medical equipment in Ethiopia. Med Biol Eng Comput 32:131-137

4. Coe G, Banta D (1992) Health care technology transfer in Latin America and the Caribbean. Int $\mathrm{J}$ Technol Assess Health Care 8:255-267

5. Engineering World Health (2010) Cambodia medical equipment inventory report, Durham, Feb 2011

6. Erinosho OA (1991) Health care and medical technology in Nigeria. Int J Technol Assess Health Care 7:545-552

7. Frize M, Cheng M (1994) Technical services for healthcare facilities: a model for developing countries. Med Biol Eng Comput 32:335-337

8. International Aid (2005) Aceh province medical equipment inventory and assessment report: Indonesia. International Aid, Spring Lake

9. Malkin RA (2007) Design of health care technologies for the developing world. Ann Rev Biomed Eng 9:567-587
10. Malkin R, Keane A (2010) Evidence-based approach to the maintenance of laboratory and medical equipment in resourcepoor settings. Med Biol Eng Comput 48:721-726

11. Pan American Health Organization (1986) Health conditions in the Americas, 1981-1984. PAHO Scientific Publication \#500, Washington, DC

12. Pan American Health Organization (1987) Fortalecimiento y Desarrollo de los Servicios de Ingenieria y Mantenimiento de Unidades de Salud. Cooperacion Tecnica, Paises Area Andina. Proyecto Subregional, Informacion Basica. Anexo I. PAHO, Oct 1987

13. Pan American Health Organization (1988) Los Servicios de Salud en Las Americas: analysis de Indicadores Basicos. Cuaderno Tecnico \#14, Washington, DC, 1388, PAHO

14. Pan American Health Organization (1993) Maintenance of Hospital Equipment. In: 21st Meeting of PAHO executive committee of the directing council, Washington, DC, 9-10 Dec 1993, SPP21/4, 8 Nov 1993

15. Pena-Mohr J (1987) Distributing and transferring medical technology a view from Latin America and the Caribbean. Int $J$ Technol Assess Health Care 3(2):281-291

16. World Bank (1994) Infrastructure and equipment. In: Better health in Africa: experience and lessons learned, The World Bank, Washington, DC, 1994 